\title{
A ticking time bomb inside the heart
}

\author{
Chandrasekaran Ananthanarayanan ${ }^{1}$, Rahul Singh ${ }^{2}$, Kartik Patel ${ }^{2}$, Archit Patel $^{2}$, Chirag \\ Doshi $^{2}$, Vivek Wadhawa ${ }^{2}$, Ramesh Patel $^{3}$, Megha Sheth ${ }^{1}$, and Pratik Shah ${ }^{1}$ \\ ${ }^{1}$ U. N. Mehta Institute of Cardiology and Research Center \\ ${ }^{2}$ U.N.Mehta Institute of Cardiology and Research Center \\ ${ }^{3}$ U.N. Mehta Institute of Cardiology and Research Center
}

September 24, 2020

\begin{abstract}
Thrombus formation inside the left ventricle (LV) is a dreaded complication following myocardial infarction. Depending on their anatomical characteristics, they pose significant risk of embolism, specifically stroke. Cardiac surgery in a patient with acute cerebral infarct is a tough predicament for the treating surgeon. Mobile clots carry higher risk than mural clots and need urgent thrombectomy. We present a case of massive LV clot in a young man leading to multiple acute embolic infarcts who was successfully treated by LV thrombectomy.
\end{abstract}

\section{Introduction:}

Even though a frequent complication of the past, thrombus formation inside the left ventricle (LV) is still reported after episodes of myocardial infarction (MI). These clots have high embolic potential with grave consequences specifically when they are mobile and huge. ${ }^{1}$ We present a case of massive LV clot in a young man leading to multiple acute embolic infarcts who was successfully treated by LV thrombectomy.

\section{Clinical Profile:}

A 34 years old male presented to our hospital with two episodes of syncope. On evaluation he was found to have acute embolic infarcts in multiple areas of his brain (Figure - $1 \mathrm{~A}-\mathrm{C}$ ). His neurological examination was normal and he gave a history of anterior wall myocardial infarction three years ago which was thrombolysed. Echocardiography showed severe Left Ventricular (LV) dysfunction with an ejection fraction of $30 \%$ and a dilated LV cavity. There was a massive freely mobile clot in the LV attached to the apex, protruding into the left ventricular outflow tract (LVOT) and hitting the aortic valve with every systole (Figure -2 A \& supplementary video). There was no evidence of LV aneurysm and the clot occupied a major part of the LV cavity. Computed tomography confirmed the echocardiographic findings (Figure -2 B). On coronary angiography, the left anterior descending artery was completely recanalized and other vessels were minimally diseased. As the clot was huge and freely mobile with high risk of further embolic showers, we planned LV thrombectomy in spite of his acute stroke.

Cardio-plumonary-bypass was established with aorto-bi-caval cannulation. After achieving cardioplegic arrest of the heart, aorta was opened. With, minimal retraction of the aortic valve leaflets, the clot was found in its entirety within the cavity. The apical attachment of the thrombus was disconnected and the clot was removed completely (Figure $-2 \mathrm{C}, \mathrm{D}$ ). Apical endocardium was found free of scars and the LV cavity was washed thoroughly with saline to flush any residues. He recovered uneventfully and his postoperative echocardiography confirmed an LV cavity free of any clots (Figure - 3A). His pre-discharge CT brain showed reduction in size of the infarcts and ruled out haemorrhagic transformation (Figure - 3B - D). 
His haematological work-up was normal and the surgical specimen was found to contain elements of old clot on histopathology. He is neurologically normal and is on regular follow up.

\section{Discussion:}

LV clot is not an uncommon, but serious complication following myocardial infarction. Data from the pre-thrombolytic era reports an incidence as high as $60 \%$ in autopsy specimens. Early myocardial revascularization has greatly reduced the incidence of LV clot and their current incidence varies between $15-30 \%$ depending upon the time of evaluation after the myocardial infarction (MI). ${ }^{2}$ They occur most commonly after anterior and apical infarcts than others. We believe a hypo/akinetic apex forms a better nidus for blood to stagnate and clot than the inflow and outflow portions with constant streaming of blood. Though LV clot is commonly caused by MI, it is also caused by pathologies such as dilated cardiomyopathy, myocarditis, takotsubo cardiomyopathy, ventricular myxoma and hypercoagulable states such as protein $\mathrm{S}$ and $\mathrm{C}$ deficiency and anti-phospholipid antibody syndrome. ${ }^{3}$ LV clots are mainly of two types - mural or protruding. Mural clots are flat and parallel to the endocardial surface with broader base. Protruding clots are pedunculated and highly mobile with a small stalk attaching them to the LV apex and they have high propensity to embolize. Embolization is the dreaded complication of LV clots with an annual incidence of around $10 \%$ and carrying more than thrice the risk of mortality compared to similar patients without LV clots. ${ }^{4}$ The management of LV clot is still murky and there are no consensus guidelines yet. It is generally accepted that huge mobile clots warrant early removal and small sessile ones can be managed conservatively with anti-coagulation. Though small clots have been documented to resolve over time, huge and mobile clots carry significant embolic risks. ${ }^{5}$ Our patient had a massive clot occupying majority of the LV with a tail hitting the aortic valve and posing a serious risk for imminent second embolic event. His scenario was further complicated by acute multiple brain infarcts. Open heart operation in acute infarcts is another grey area within the cardiac surgical community and we don't have a guideline-based management protocol till date. Pre-operative acute stroke is a known risk factor for cardiac surgery. But there are many published reports proving the safety of cardiac surgery in acute embolic infarcts. ${ }^{6}$ We believe that unless the patient is moribund or having a massive infarct, acute embolic stroke should not defer open heart surgery specifically in patients with heavy embolic load like ours. Surgical approach for LV thrombectomy is varied. Concomitant procedures such as mitral valve or LV aneurysm repair dictates the approach most of the times. Ventriculotomy is better avoided for isolated LV thrombectomy as an incision in the LV wall can promote arrhythmogenicity in an already dysfunctional heart and will make future ventricular assist device insertions difficult if required. ${ }^{7}$ Trans-mitral approach can be used when the clot is small and limited to the apex, but can prove difficult in massive clots protruding into the LVOT like ours. ${ }^{8}$ Trans-aortic approach is very simple and effective for LV thrombectomy as the aortic valve and LVOT are anatomically in line with the apex and a dilated apex in majority of these patients enhances the visualization further. Organised clots can be approached by a combined trans-mitral and trans aortic route to ensure complete thrombus removal and a thorough wash of the LV cavity from both its inlet and the outlet to catch residues if any.

\section{Conclusion:}

Massive mobile LV clots are literally ticking time bombs inside the heart and needs urgent removal to prevent further embolic catastrophes even in acute stroke, unless the patient is in extremis with a massive infarct. Trans-aortic approach is a simple and effective method for LV thrombectomy.

\section{Acknowledgement:}

The authors gratefully acknowledge Mr Ashish Patel for assistance.

\section{References}

1. Leick J, Szardien S, Liebetrau C, et al. Mobile left ventricular thrombus in left ventricular dysfunction: case report and review of literature. Clin Res Cardiol. 2013;102(7):479-84.

2. Solheim S, Seljeflot I, Lunde K, et al. Frequency of left ventricular thrombus in patients with anterior wall acute myocardial infarction treated with percutaneous coronary intervention and dual antiplatelet 
therapy. Am J Cardiol. 2010 Nov 1;106(9):1197-200.

3. Forkmann M, Tugtekin SM, Strasser RH, Schrötter H. Impending paradoxical thromboembolism: Thrombus caught in transit. A case report. Clinical Research in Cardiology. 2012;101(6):497-8.

4. Fruster V, Halperin JL. Left ventricular thrombi and cerebral embolism. N Engl J Med 320(6):392-394.

5. Mallory R, Balcezak T. Treatment of mobile left ventricular thrombus with low-molecular-weight heparin. N Engl J Med. 1999;341(14):1082-3.

6. Ananthanarayanan C, Menon S, Dharan B, Mathew T, Cherian VT, Koshy T. Acute cardio-embolic stroke in a preschooler - a surgical dilemma.

7. Tsukube T, Okada M, Ootaki Y, Tsuji Y, Yamashita C. Transaortic video-assisted removal of a left ventricular thrombus. Ann Thorac Surg. 1999; 68(3):1063-5.

8. Early GL, Ballenger M, Hannah III H, Roberts SR. Simplified method of left ventricular thrombectomy. Ann Thorac Surg. 2001; 72(3):953-4.

\section{Figure legends:}

1. Computed Tomographic images of the brain showing infarcts in the following areas A. right temporoparietal B. right thalamus, right occipital C. left cerebellum

2. A. Intra-operative trans-oesophageal echocardiographic image showing a massive left ventricular clot hitting the aortic valve in systole B. Computed tomographic image showing the huge clot occupying major part of the left ventricular cavity. C. Intra-operative images showing surgical removal of the thrombus D. Thrombectomy specimen

3. A. post-operative trans-oesophageal echocardiography showing left ventricular cavity free of thrombus, (B-D) pre-discharge computed tomographic images of the brain showing reduction in size of the infarct and absence of haemorrhagic transformation in the following areas of the brain B. right temporoparietal C. right thalamus, right occipital D. left cerebellum

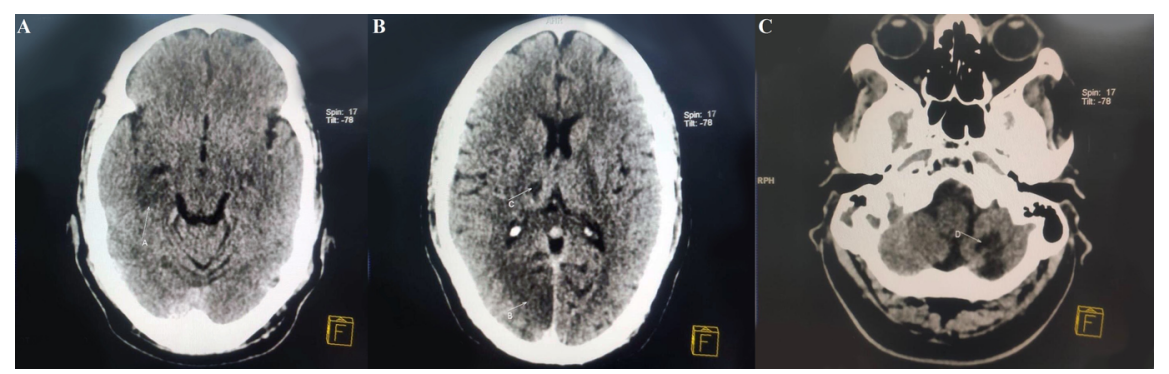



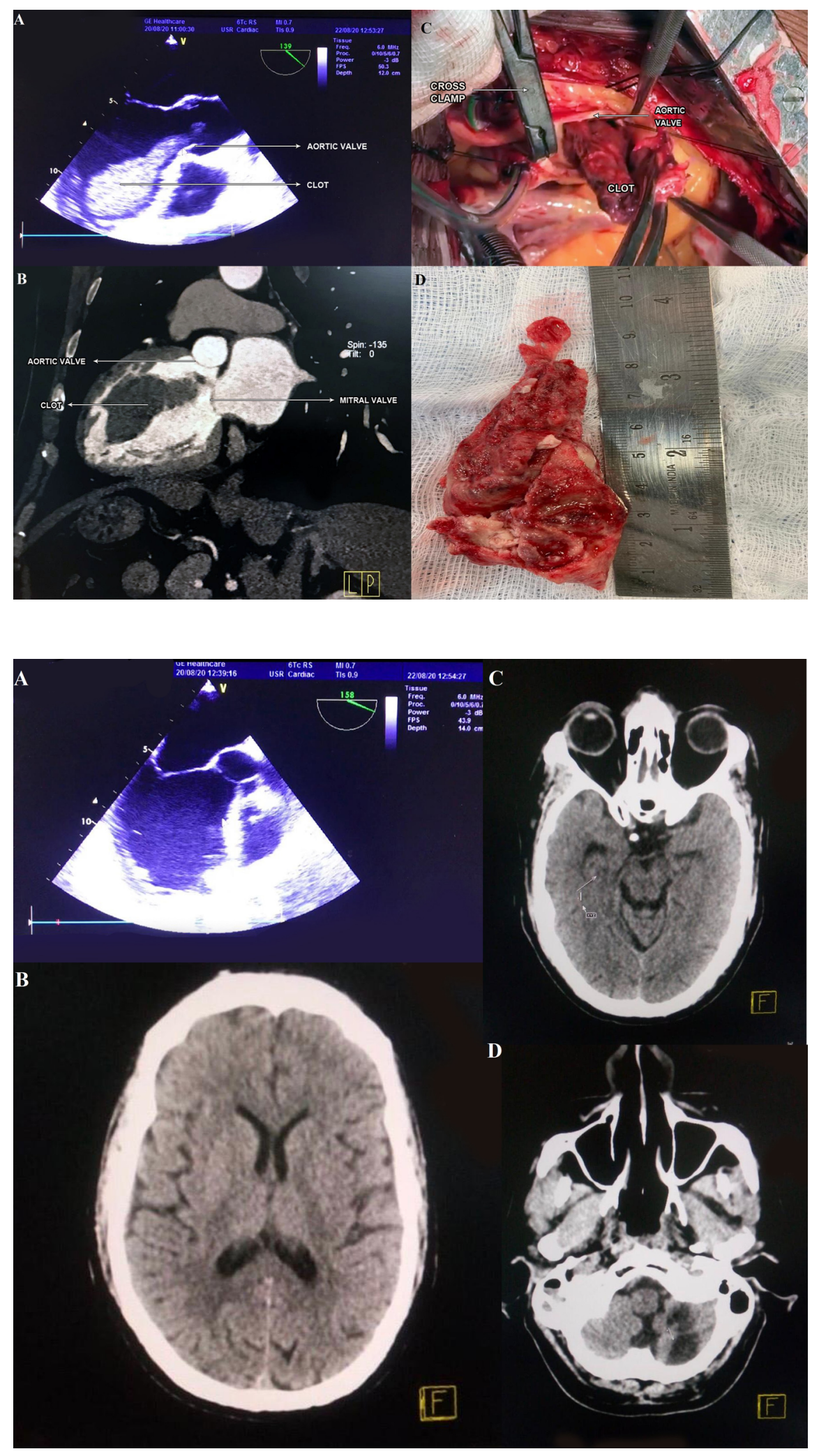